\title{
Associações entre Informações, Desempenho da Controladoria, Desempenho Gerencial e Organizacional: um Estudo Exploratório
}

\begin{abstract}
Resumo
Os livros-textos afirmam que a área de Controladoria é importante para a tomada de decisão e gestão, e muito embora existam argumentos teóricos favoráveis a considerar o controle gerencial como um recurso que influencia o desempenho organizacional (Henri, 2006), ainda há uma grande carência de estudos empíricos que identifiquem a contribuição da área de Controladoria para o desempenho das organizações. Este artigo se propôs a identificar os relacionamentos entre fontes de informações ( $M o l \&$ Birknshaw, 2009), desempenho da controladoria, desempenho gerencial e desempenho organizacional, com base em 80 respostas de profissionais de controladoria e finanças de empresas brasileiras. Os resultados, analisados pela técnica estatística Modelagem de Equações Estruturais, indicam que existem associações positivas entre grau de obtenção de informações e desempenho da controladoria, e também que este último afeta positivamente o desempenho gerencial, com subsequente efeito positivo no desempenho organizacional. Os resultados sugerem que uma área de Controladoria, que busca conhecimento nas fontes internas e externas, consegue gerar informações com boa qualidade e no tempo adequado, a um custo compatível e focada em projetos, e consegue atender às expectativas dos usuários. Esses gestores, por sua vez, suportados por uma Controladoria eficaz e eficiente, planejam, controlam e tomam decisões mais assertivas, contribuindo para que a organização atinja satisfatoriamente o principal objetivo da organização. O estudo inova ao relacionar variáveis mais ligadas ao papel da controladoria, além de operacionalizar o construto desempenho da controladoria, com base na visão de acadêmicos e profissionais, mas tem como principal limitação o fato de ser baseado na percepção dos próprios profissionais da controladoria.
\end{abstract}

Palavras-chave: Controladoria; Desempenho; Informações; Desempenho da Controladoria

\begin{abstract}
José Carlos Tiomatsu Oyadomari Doutor em Controladoria e Contabilidade pela FEA/USP e Professor Adjunto Universidade Presbiteriana Mackenzie UPM. Contato: Rua da Consolação, 896, CCSA - Prédio T - NECO - Sala 602, Consolação, São Paulo, SP. CEP: 01302-907. E-mail: oyadomari@mackenzie.br
\end{abstract}

\section{Andson Braga de Aguiar} Doutor em Controladoria e Contabilidade pela FEA/USP e Professor da Universidade de São Paulo. Contato: Av. Prof. Luciano Gualberto, 908, Complemento: FEA3, Sala 220, Butantã, São Paulo, SP. CEP: 05508-010.

E-mail: abraga@usp.br

\section{Chen Yen-Tsang}

Doutorando em Administração de Empresas pela EAESP/FGV. Contato: Av. Nove de Julho, 2029. POI, Bela Vista, São Paulo, SP. CEP: 01313-001. E-mail: yentsang@gvmail.br

\section{Ricardo Lopes Cardoso} Doutor em Controladoria e Contabilidade pela FEA/USP e Professor Adjunto da Universidade Presbiteriana Mackenzie UPM. Contato: Rua da Consolação, 896, CCSA - Prédio T - NECO - Sala 602, Consolação, São Paulo, SP. CEP: 01302-907. E-mail: ricardo.cardoso@mackenzie.br

\section{Ronaldo Gomes Dultra-de-Lima} Doutorando em Administração de Empresas EAESP/FGV e Professor Assistente da Universidade Presbiteriana Mackenzie. Contato: Rua da Consolação, 896, CCSAPrédio T-Sala dos professores, Consolação, São Paulo, SP. CEP: 01302-907 E-mail: ronaldo.lima@gvmail.br 


\section{Introdução}

Há consenso que o presente estágio da sociedade se caracteriza pelo conhecimento e que, para melhorar o desempenho, as organizações devem desenvolver modelos que permitam adquirir, compartilhar e disseminar o conhecimento para toda a organização (Nonaka \& Takeuchi, 1997). Na área contábil, nota-se que os estudos que têm discutido este tema, conhecido como gestão do conhecimento, estão focados em empresas de consultoria e auditoria (Morris \& Empson, 1998, Vera-Muñoz, Ho \& Chow, 2006), deixando de lado a área de Controladoria, o que não se justifica, uma vez que esta como qualquer unidade organizacional também adquire conhecimento, e os estudos comprovam que o conhecimento influencia positivamente o sucesso dos profissionais de controladoria (Hunton, Wier \& Stone, 2000).

Do ponto de vista das pesquisas organizacionais e considerando que o ambiente de trabalho dos profissionais da área Contábil/Controladoria ocorre nos departamentos de Controladoria, é, portanto, mais apropriado que se meça o desempenho na perspectiva organizacional, isto é, pelo desempenho da área de Controladoria, em vez de medir o sucesso individual, o que é coerente com a maior preocupação com a medição de desempenho de áreas funcionais (Chenhall \& Langfield-Smith, 2007).

Mas, se justificada a questão de medir o desempenho da área de Controladoria, outra discussão ainda permanece: Qual a real contribuição da área de Controladoria para o desempenho organizacional? Os livros-textos são categóricos em afirmar que essa área é importante para a gestão de uma organização (Catelli,1999, Garrison, Noreen \& Brewer, 2007). Por outro lado, os estudos empíricos estão mais focados em identificar as funções da área de Controladoria (Borinelli, 2006, Lunckes, Schnorrenberger \& Rosa, 2013), em detrimento de identificar o desempenho da própria área e os relacionamentos com o desempenho organizacional, constituindo-se em uma lacuna de pesquisa.

Por outro lado, é sabido que as funções da área de Controladoria consolidadas pela literatura são planejamento, controle, elaboração e interpretação de relatórios (Lunckes, Schnorrenberger \& Rosa, 2013), e que o desempenho da área de Controladoria influencia o processo de tomada de decisão pelos gestores das áreas-fins da organização (Weibenberger \& Angelkort,2011). Assim, não é recomendável relacionar de forma direta o desempenho da área de Controladoria com o desempenho organizacional.

Essa ligação indireta com o desempenho organizacional é coerente com a perspectiva da ResourcesBased View, onde o Sistema de Controle Gerencial pode ser classificado como um recurso, desde que utilizado complementarmente a outros recursos (Barney, 2007), tese que também é compartilhada por Henri (2006).

Estudar os departamentos se justifica, pois os estudos em estratégia têm sugerido que a vantagem competitiva pode existir no nível dos processos, ou seja, nas rotinas ou atividades desenvolvidas pela empresa, e que o desempenho destas não necessariamente pode ser refletido no desempenho geral da empresa (Ray, Barney \& Muhanna, 2004). Uma dessas rotinas é o processo de gerenciamento, que pode ser medido pelo construto "desempenho gerencial" consoante os estudos de Robertson, Baron, Gibbons, Maciver e Nyfield (2000) e Craik, Ware, Kamp, O’Reilly, Staw e Zedeck (2002), o qual abrange uma lista que inclui oito dimensões de atividades gerenciais (Tsui, 2001).

Há, portanto, uma oportunidade de pesquisa que possa estudar quais são as fontes de informação utilizadas pela área de Controladoria, e como estas se associam com o desempenho da própria área, em uma tentativa de validar estudos normativos sobre o papel da área. Adicionalmente, faltam trabalhos que relacionem o desempenho da área de Controladoria com o desempenho gerencial, uma vez que a área de Controladoria subsidia os gestores com informações para a tomada de decisão, planejamento e controle. Por fim, em uma perspectiva utilitarista, há uma oportunidade de identificar se o relacionamento dessas variáveis contribuem para que a organização atinja o principal objetivo, que é uma forma de operacionalizar o desempenho organizacional.

Considerando essas fundamentações, este trabalho se propõe a responder a seguinte questão de pesquisa: Quais são os relacionamentos entre fontes de Informação, Desempenho da Controladoria, Desempenho Gerencial e Desempenho Organizacional? 
As contribuições esperadas deste trabalho para a literatura acadêmica se constituem em estudar um tema ainda pouco estudado, pois não se encontrou nenhum trabalho que estudasse todos esses construtos, nem mesmo internacionalmente relacionados com a área de Controladoria, e com a possibilidade de operacionalizar construtos mais relacionados com a área de Controladoria. Para o campo da prática, espera-se que o estudo contribua ao fornecer um modelo que relacione as principais variáveis que tenham impacto positivo nas diferentes dimensões de desempenho (gerencial e organizacional).

\section{Referencial Teórico}

\subsection{Fontes de Obtenção de Informação}

Uma tipologia adotada é a que classifica as fontes de obtenção de informações em internas, de mercado e profissionais. Mol e Birkinshaw (2009) utilizaram e detalharam essas categorias em Internas (dentro da empresa, outras empresas do mesmo grupo), Mercado (Fornecedores de equipamento, materiais, componentes ou softwares, Clientes ou consumidores, Concorrentes, Consultores, Laboratórios Comerciais / Empresas de Pesquisa Desenvolvimento), Profissionais (Conferências e reuniões Profissionais, Associações comerciais, Revistas Técnicas, bases de dados, Feiras e Exposições). Essa tipologia de Mol e Birkinshaw (2009) é coerente com o que propõem Nonaka e Takeuchi (1997), quando estes discutem que uma das etapas da socialização é a captura de conhecimento tanto de fontes externas como em fontes internas.

Em linhas gerais, Mol e Birkinshaw (2009) argumentam que quanto mais fontes de informação utilizadas pela empresa, maior é o grau de introdução de novas práticas gerenciais. Por outro lado, há que se considerar que o paradoxo existente é caracterizado por uma crescente quantidade de informações com concomitante atenção limitada (Simons, 2000).

Diversos estudos relacionam aspectos do Modelo de Nonaka \& Takeuchi com desempenho organizacional, mas sempre mediados por algum construto intermediário. Ramirez, Morales e Rojas (2011) estudaram o modelo de Nonaka e Takeuchi (1997) e argumentam que a combinação (captura e integração de novos conhecimentos explícitos essenciais, por meio de coleta, reflexão, e síntese) pode melhorar a internalização desses conhecimentos. Os resultados mostraram que o processo de criação e compartilhamento de conhecia influencia direta ou indiretamente a aprendizagem organizacional, e que esta influencia positivamente o desempenho organizacional (Ramirez, Morales \& Rojas, 2011).

\subsection{Desempenho da Área de Controladoria}

A área de Controladoria, como qualquer área organizacional, também necessita obter uma avaliação de desempenho favorável em relação aos diversos objetivos traçados, como, por exemplo, atendimento de prazos, cumprimento do orçamento da área, qualidade nas informações fornecidas, entre outras. Muito embora o desempenho possa ser medido pelo grau de participação do controller no planejamento estratégico (Cadez \& Guilding, 2008), esse construto ainda não abrange o desempenho da área funcional. Assim medidas de desempenho específicas têm sido desenvolvidas para atender a necessidades de gestão de diversas funções, como Operações, Marketing ou Recursos Humanos (Chenhall \& Langfield-Smith, 2007).

Para a área de Controladoria, pode-se identificar a proposta por Mouritsen (1996), cujo autor estudou cinco atividades desempenhadas pelo departamento de Contabilidade, como Registro, Consultoria, Gestão Financeira, Controle e Gestão Administrativa, porém, sem a preocupação de refletir essas atividades como desempenho. Já no estudo de Tsui (2001), o construto Sistemas de Contabilidade Gerencial foi mensurado com nove perguntas, que podem ser entendidas também como desempenho da área de Controladoria, uma vez que as variáveis abrangeram itens, como pontualidade dos relatórios, 
informações preditivas, informações não monetárias, rapidez no fornecimento de informações, estimativas de probabilidade, tempestividade, informações não monetárias mais relacionadas às competências e atitudes, informações sobre variáveis estratégicas como tecnologia, economia, etc.

Baseados em Hunton et al. (2000), para quem o conhecimento impacta positivamente o desempenho, e que o processo de gestão de conhecimento aplicado à área de Controladoria passa pela aquisição de informações, e em Ramirez, Morales e Rojas (2011), os quais justificaram o relacionamento positivo entre obtenção de informações, aprendizagem organizacional e desempenho, fundamenta-se a seguinte Hipótese 1: Grau de Obtenção de informações está associado positivamente com o Desempenho da Controladoria

\subsection{Desempenho Gerencial}

Diversas competências organizacionais têm sido estabelecidas como importantes, mas muitas delas podem ser peculiares a determinadas organizações, porém, um processo ou uma rotina comum a todas elas é o processo gerencial, o qual pode ser caracterizado como uma rotina (Ray et al., 2004). Uma forma de medir o processo gerencial pode ser por meio do desempenho gerencial, o qual tem sido fruto de estudos no campo da psicologia (Robertson et al., 2000; Craik et al., 2002), e mais recentemente no campo da Contabilidade Gerencial (Tsui, 2001; Agbejule \& Saarikoski, 2006). De forma geral, pode-se dizer que esses estudos têm se baseado no construto desenvolvido por Mahoney, Jerdee e Carroll (1965).

Tsui (2001) estudou os relacionamentos entre participação orçamentária, sistemas de contabilidade gerencial e desempenho gerencial, analisando o impacto das culturas chinesa e ocidental. Os resultados mostraram que a participação orçamentária afetava negativamente o desempenho gerencial nas empresas chinesas, enquanto ocorria o contrário nas empresas ocidentais.

Já Agbejule e Saarikoski (2006), com base em um survey com 83 gerentes de empresas finlandesas, identificaram que quanto maior a participação orçamentária e maior o nível de conhecimento de gestão de custos, maior é o desempenho gerencial, medido por autopercepção. Nesse estudo, o desempenho gerencial foi medido por oito variáveis: Representação, Negociação, Investigação, Avaliação, Supervisão, Planejamento, Coordenação e Apoio, sendo que, após a análise fatorial, este construto foi agrupado em dois: desempenho gerencial externo, o qual agrupou representação e negociação e os demais, com exceção de investigação que foi eliminado, como desempenho gerencial interno.

Em linha com estes estudos de Contabilidade Gerencial, mas com o propósito de identificar variáveis mais relacionadas com a área de Controladoria, identificou-se que dois principais propósitos da Contabilidade para o processo gerencial são planejar e controlar os recursos, o que está de acordo com Malmi e Grandlund (2009), para quem as informações contábeis gerenciais servem para planejar, avaliar, controlar e assegurar o uso apropriado e a responsabilidade pelos seus recursos. Outro propósito da Contabilidade Gerencial é a tomada de decisões, o que também é coerente com o estudo de Weibenberger e Angelkort (2011), que mediu a influência do controller nas decisões gerenciais e também consoante a afirmação de Hall (2010), para quem a informação contábil provida na forma de relatórios é a base para tomada de decisões pelos gerentes.

Tendo como base essas fundamentações, declara-se a seguinte hipótese Hipótese 2: Desempenho da Controladoria associa-se positivamente com Desempenho Gerencial.

\subsection{Desempenho Organizacional}

O desempenho organizacional pode ter um significado bastante amplo, que pode incluir indicadores monetários, como eficiência e lucratividade, ou não monetários, como satisfação dos empregados e taxa de inovação (Donaldson, 2001). 
Crossan, Rouse, Fry e Killing (2009) também afirmam que, para evitar os conflitos e a confusão entre os diferentes objetivos organizacionais, estes devem ser postos em alguma ordem de prioridade, também chamada de estrutura de objetivos. Na mesma linha, Anthony e Govindarajan (2006, p.88) enfatizam que uma organização tem diferentes objetivos, mas afirmam que "no sistema formal de controle gerencial de uma empresa, a lucratividade é geralmente, o objetivo mais importante". Já Hitt, Ireland e Hoskisson (2001) discutem que uma empresa, atingindo competitividade estratégica e explorando a sua vantagem competitiva, se habilita para alcançar o seu objetivo primário que é retornos de lucros acima da média.

Nesse sentido o Desempenho Organizacional neste trabalho é operacionalizado como o grau de atingimento da principal meta da organização, consoante a afirmação destacada na literatura de que há uma escala de prioridade de objetivos. Embora se reconheça que a indicação de uma única variável para medir este construto possa ser uma limitação, essa escolha é deliberada para evitar potenciais variabilidades que poderiam ocorrer com a inclusão de diversas variáveis.

Os estudos que adotam a perspectiva teórica Resources-Based View têm adotado relações causais entre competências e desempenho. Neste caso, a ideia principal é que o desempenho é mais influenciado pelos recursos e competências. Uma competência é, em essência, uma rotina ou uma série de rotinas que se interagem (Grant, 1991; Barney, 2001), e uma dessas rotinas organizacionais é o processo gerencial aqui operacionalizado como Desempenho Gerencial. Dessa forma, fundamenta-se a terceira hipótese Hipótese 3: Desempenho Gerencial associa-se positivamente com o Desempenho Organizacional.

\section{Procedimentos Metodológicos}

Classifica-se esta pesquisa como exploratória, uma vez que os estudos ainda podem ser considerados em estágios iniciais, principalmente em relação aos construtos Desempenho da Controladoria e Desempenho Gerencial. Os estudos exploratórios são particularmente importantes em um momento em que há questionamentos sobre as contribuições das pesquisas acadêmicas para o campo dos práticos (Baldvinsdottir, Mitchell \& Norreklit, 2010). Dessa forma, esta pesquisa é exploratória, descritiva e com método quantitativo de análise (Hair Jr., Anderson \& Black, 2005; Flick, 2009).

\subsection{Amostra}

Com o objetivo de garantir melhor entendimento sobre as questões, foi feito um pré-teste (Dillman, 2000) com um diretor financeiro de uma indústria de autopeças e dois professores doutores com experiência em pesquisas do tipo survey. Pequenas alterações de redação foram feitas sem que houvesse mudança nos construtos.

A população considerada foi a de gerentes de Controladoria, gerentes financeiros, gerentes de contabilidade de empresas brasileiras. Porém, dado que não existe uma base unificada de empresas que abranja empresas de diferentes portes, como é a realidade das empresas brasileiras, optou-se por desenvolver uma base que possa representar de forma mais adequada a população de empresas brasileiras. Dessa forma, optou-se por construir uma base abrangendo (1) Cadastro de respondentes originados de pesquisas anteriores; (2) Cadastro de gerentes de Controladoria de uma empresa de consultoria e treinamento; (3) Membros de um grupo de rede social focado em Controladoria e Contabilidade. A escolha de membros de rede social é justificada em função de que essas redes auxiliam na produção e disseminação de conhecimento e inovações relacionadas ao mundo corporativo (Martins, Quincozes, Pereira \& Fialho, 2009).

Portanto, a despeito de ser construída por conveniência como grande parte das pesquisas brasileiras em contabilidade gerencial, entende-se que essa amostra reflete com melhor propriedade o universo de gestores que atuam em empresas brasileiras, uma vez que abrange gestores que atuam em médias empresas, as quais não divulgam dados contábeis. 
A pesquisa foi desenvolvida durante os meses de novembro e dezembro de 2010 e o questionário foi enviado por correspondência eletrônica, por meio de um serviço de disponibilização de questionário de forma eletrônica.

\subsubsection{Informações Descritivas sobre os Respondentes}

A Tabela 1 e Tabela 2 fornecem informações descritivas sobre os respondentes, enquanto que a Tabela 3 permite o entendimento do porte das empresas em que tais respondentes atuam. Com base nas informações contidas nessas tabelas, pode-se considerar que os respondentes atendem ao perfil desejado para a realização dessa pesquisa, tendo em vista os objetivos propostos.

Tabela 1

Respondentes por Departamento

\begin{tabular}{lcc}
\multicolumn{1}{c}{ Departamento } & Quantidade & Percentagem \\
\hline Centro de Serviços Compartilhados & 2 & $2,5 \%$ \\
\hline Contabilidade & 10 & $12,5 \%$ \\
\hline Controladoria & 50 & $62,5 \%$ \\
\hline Custos e Orçamentos & 6 & $7,5 \%$ \\
\hline Financeiro & 12 & $15,0 \%$ \\
\hline Total & 80 & $100,0 \%$ \\
\hline
\end{tabular}

Fonte: Dados da pesquisa

Tabela 2

Respondentes por cargo

\begin{tabular}{|c|c|c|}
\hline Cargo & Quantidade & Percentagem \\
\hline Chefe & 1 & $1,3 \%$ \\
\hline Coordenador & 15 & $18,8 \%$ \\
\hline Diretor & 12 & $15,0 \%$ \\
\hline Gerente & 41 & $51,3 \%$ \\
\hline Gerente Geral & 6 & $7,5 \%$ \\
\hline Supervisor & 5 & $6,3 \%$ \\
\hline Total & 80 & $100,0 \%$ \\
\hline
\end{tabular}

Fonte: Dados da pesquisa 
Tabela 3

Porte das empresas

\begin{tabular}{|c|c|c|c|c|}
\hline \multicolumn{3}{|c|}{$\begin{array}{c}\text { Intervalo de Classes em } \\
\text { Quantidade de Empregados }\end{array}$} & \multirow{2}{*}{$\begin{array}{c}\text { Quantidade de empresas } \\
11\end{array}$} & \multirow{2}{*}{$\begin{array}{r}\text { Percentagem } \\
13,75 \% \\
\end{array}$} \\
\hline [ & $0 ; 100$ & ] & & \\
\hline[ & $101 ; 500$ & ] & 18 & $22,50 \%$ \\
\hline[ & $501 ; 1.000$ & ] & 14 & $17,50 \%$ \\
\hline[ & $1.001 ; 5.000$ & ] & 24 & $30,00 \%$ \\
\hline[ & $5.001 ; 10.000$ & ] & 9 & $11,25 \%$ \\
\hline[ & Acima de 10.000 & ] & 4 & $5,00 \%$ \\
\hline Total & & & 80 & $100,00 \%$ \\
\hline
\end{tabular}

Fonte: Dados da pesquisa

\subsection{Elaboração do questionário}

\subsubsection{Fontes de obtenção de conhecimento}

Muito embora a tipologia desenvolvida por Mol e Birkinshaw (2009) seja importante, entende-se que ela foi desenvolvida para inovações organizacionais de forma abrangente, sendo necessário introduzir alguns itens mais aplicáveis ao departamento de Controladoria. Nesse sentido, três variáveis foram incluídas para fins deste estudo:

- Livros - Os livros têm sido apresentados como importantes no papel da institucionalização das práticas de contabilidade gerencial (Scapens, 1994).

- Artigos acadêmicos - Van Helden, Aardema, Ter Bogt e Groot (2010) identificaram que pesquisadores buscam esse conhecimento em periódicos acadêmicos internacionais, enquanto que os consultores obtêm conhecimento por meio de contatos com colegas e em revistas profissionais. Embora haja essa dissonância, essa variável foi incluída na tentativa de aferir a relevância da produção acadêmica para a prática, uma preocupação manifestada por diversos autores como Malmi e Grandlund (2009), e Baldvinsdottir, Mitchel e Nørreklit (2010).

- Rede de contatos - Calhoun e Starbuck (2003) fornecem uma explicação dos relacionamentos entre informação e conhecimento. Para eles, as pessoas e as organizações adquirem informação por meio de redes sociais, e uma porção dessa informação se torna conhecimento (Calhoun \& Starbuck, 2003). Pettigrew e Massini (2003) também destacam o papel do compartilhamento de informação, além das fronteiras organizacionais.

As demais variáveis foram adaptadas do estudo de Mol e Birkinshaw (2009).

Os respondentes tinham que indicar a importância de cada uma das principais fontes de conhecimento ou informação utilizadas para gerar inovações nas técnicas de controle gerencial, em um total de nove itens, tendo como base os últimos dois anos. Escala 1. Não Utilizado, 2. Pouca Importância, 3.Média Importância, 4. Grande Importância. A Tabela 4 apresenta os dados sobre as variáveis que compõem o construto Fontes de Informação.

Para evitar repetir os dados de média e desvio-padrão, optou-se por já colocar esses dados junto com a operacionalização das variáveis. 
Tabela 4

Fontes de Informação

\begin{tabular}{lccccc}
\hline \multicolumn{1}{c}{ Fonte de informação } & Variável & $\begin{array}{c}\text { Classificação } \\
\text { segundo Mol \& } \\
\text { Birkinshaw (2009) }\end{array}$ & Média & Desvio-Padrão & Referencial \\
\hline $\begin{array}{l}\text { Informações obtidas nos } \\
\text { outros departamentos }\end{array}$ & F1 & Interna & 3,6 & 0,7 & $\begin{array}{l}\text { Adaptada de Mol e } \\
\text { Birkinshaw (2009) }\end{array}$ \\
\hline Consultores externos & F2 & Mercado & 2,8 & 0,8 & Mol e Birkinshaw (2009) \\
\hline Concorrentes & F3 & Mercado & 2,9 & 0,9 & Mol e Birkinshaw (2009) \\
\hline Livros & F4 & Profissional & 3,0 & 0,8 & $\begin{array}{l}\text { Baseado em Scapens } \\
\text { (1994) }\end{array}$ \\
\hline Fornecedores de Software & F5 & Mercado & 3,0 & 0,8 & Mol e Birkinshaw (2009) \\
\hline Jornais e Revistas de Negócios & F6 & Profissional & 3,0 & 0,7 & Mol e Birkinshaw (2009) \\
\hline Artigos Acadêmicos & F7 & Profissional & 2,8 & 0,8 & $\begin{array}{l}\text { Baseado em Van Helden } \\
\text { et al. (2010) }\end{array}$ \\
\hline $\begin{array}{l}\text { Associações de classe } \\
\text { (empresariais e profissionais) }\end{array}$ & F8 & Profissional & 3,0 & 0,9 & Mol e Birkinshaw (2009) \\
\hline $\begin{array}{l}\text { Rede de contatos } \\
\text { Seminários e Cursos }\end{array}$ & F9 & Profissional & 3,3 & 0,8 & $\begin{array}{l}\text { Calhoun e Starbuck } \\
\text { (2003) }\end{array}$ \\
\hline
\end{tabular}

Fonte: Dados da pesquisa

\subsubsection{Desempenho da Controladoria}

Embora não seja o principal objetivo deste trabalho, uma vez que um relato detalhado da operacionalização do construto Desempenho da Controladoria poderia ser objeto de artigo específico, esta seção descreve sinteticamente os principais procedimentos envolvendo este trabalho para desenvolver o construto Desempenho da Controladoria.

Um questionário foi enviado a uma amostra, definida por conveniência, abrangendo professores/ pesquisadores da área de Contabilidade Gerencial e profissionais atuantes em empresas. A amostra de acadêmicos buscou parte dos professores vinculados aos Programas de Pós-Graduação (Stricto Sensu) em Contabilidade, com envio de dez questionários e retorno de seis válidos. A amostra de profissionais foi obtida por meio de contato com profissionais vinculados à associação de profissionais de finanças e contabilidade como o Instituto Brasileiro de Executivos em Finanças (Ibef-SP) e a Associação Nacional de Executivos de Finanças, Administração e Contabilidade (Anefac), além de alguns profissionais da rede de contato dos pesquisadores, todos com cargos acima de gerente e com formação acadêmica mínima em MBA/Especialização. Nesse caso, nove questionários foram considerados de um total de dezoito questionários enviados.

Os respondentes foram solicitados a informar, por meio de questionário eletrônico por internet, sobre quais aspectos a área de Controladoria deveria ter o desempenho medido e avaliado e solicitava justificativas. Procedimento semelhante foi adotado por Frezatti, Aguiar e Guerreiro (2007) para avaliar as diferenças entre Contabilidade Financeira e Gerencial, só que envolvendo somente acadêmicos, mas de diversos países. 
Em síntese, os principais aspectos que acadêmicos e profissionais mencionaram podem ser identificados na Figura 1.

\begin{tabular}{lll}
\hline \multicolumn{1}{c}{ Variável } & \multicolumn{1}{c}{ Acadêmicos } & \multicolumn{1}{c}{ Profissionais } \\
\hline Cumprimento de prazos & $\begin{array}{l}\text { Cumprimento de prazos e Tempestividade } \\
\text { da informação }\end{array}$ & $\begin{array}{l}\text { Qualidade da informação prestada e } \\
\text { prazo }\end{array}$ \\
\hline $\begin{array}{l}\text { Qualidade das informações } \\
\text { prestadas }\end{array}$ & $\begin{array}{l}\text { Oportunidade e relevância da informação. } \\
\text { Avaliar se as informações prestadas } \\
\text { conduziram aos melhores resultados }\end{array}$ & $\begin{array}{l}\text { Pontualidade na entrega; nível de } \\
\text { erros; nível de entendimentos das suas } \\
\text { informações }\end{array}$ \\
\hline $\begin{array}{l}\text { Controle orçamentário dos } \\
\text { gastos departamentais }\end{array}$ & $\begin{array}{l}\text { Eficiência em relação aos serviços } \\
\text { prestados }\end{array}$ & $\begin{array}{l}\text { Controle das despesas do } \\
\text { departamento }\end{array}$ \\
\hline $\begin{array}{l}\text { Satisfação dos usuários } \\
\text { (clientes internos) }\end{array}$ & $\begin{array}{l}\text { Avaliar se as informações prestadas } \\
\text { conduziram aos melhores resultados }\end{array}$ & Satisfação dos usuários \\
\hline $\begin{array}{l}\text { Execução de projetos do } \\
\text { departamento }\end{array}$ & Implementação de novas ferramentas & $\begin{array}{l}\text { Planejamento e efetividade das } \\
\text { atividades sob gestão da Controladoria. } \\
\text { Execução dos projetos do } \\
\text { departamento }\end{array}$ \\
\hline
\end{tabular}

Fonte: Elaborado pelos autores com base em dados da pesquisa

Figura 1. Amarração das variáveis com evidências de acadêmicos e profissionais

A análise de conteúdo consoante proposto por Bardin (2004) mostra que os dois aspectos mais citados são cumprimento de prazos e qualidade da informação prestada. Esses são aspectos muito presentes em uma relação de prestação de serviços, sugerindo que a Controladoria é vista como um departamento prestador de serviços voltado para o atendimento das demandas de clientes internos e externos em uma tácita relação contratual.

Dois outros aspectos foram destacados: um deles foi o de a Controladoria ter como objetivo o controle dos seus próprios gastos. Isto demonstra que a Controladoria, como qualquer outra área da empresa, deve responder pelo seu orçamento de despesas. Outro aspecto considerado relevante é a questão da execução dos projetos, sendo que um dos respondentes destacou o fato de a área de Controladoria estar desenvolvendo diversos projetos de como adotar as novas normas contábeis. Por fim, a satisfação dos usuários é uma preocupação tanto de acadêmicos como de usuários, sendo que os acadêmicos destacaram o aspecto da tomada de decisão.

Dessa forma, após análise de conteúdo consoante sugerido por Bardin (2004), os seguintes itens foram considerados: (1) Cumprimento de prazos; (2) Qualidade das informações prestadas; (3) Controle orçamentário dos gastos departamentais; (4) Satisfação dos usuários (clientes internos); (5) Execução de projetos do departamento. Dessa forma grande parte do desempenho, exceto o item 4, está alinhada com o que Borinelli (2006) chamou de Estrutura Conceitual Básica de Controladoria em sua perspectiva "aspectos procedimentais", que são as atividades, funções e artefatos utilizados.

Os respondentes foram solicitados a indicar o grau de cumprimento em relação aos objetivos do departamento de Controladoria, sendo utilizada uma escala Likert de "1. Muito Abaixo do Esperado" a "7. Muito Acima do Esperado". A Tabela 5 apresenta os dados relativos ao construto Desempenho de Controladoria. 
Tabela 5

Variáveis Desempenho da Controladoria

\begin{tabular}{lcccc}
\multicolumn{1}{c}{ Assertiva } & Variável & Média & Desvio Padrão & Fonte \\
\hline Cumprimento de prazos & DC1 & 5,4 & 1,2 & Pesquisa \\
\hline Qualidade das informações prestadas & DC2 & 5,7 & 0,9 & Pesquisa \\
\hline $\begin{array}{l}\text { Controle orçamentário dos gastos } \\
\text { departamentais }\end{array}$ & DC3 & 5,3 & 1,3 & Pesquisa \\
\hline Satisfação dos usuários (clientes internos) & DC4 & 5,3 & 1,1 & Pesquisa \\
\hline Execução de projetos do departamento & DC5 & 5,1 & 1,3 & Pesquisa \\
\hline
\end{tabular}

Fonte: dados da pesquisa

\subsubsection{Desempenho Gerencial}

A mensuração do Construto abrangeu três variáveis (planejamento das atividades, controle dos recursos e tomada de decisão). Os respondentes foram solicitados a indicar como eles avaliam os gestores em relação a algumas atividades, sendo utilizada uma escala Likert de "1. Muito Abaixo do Esperado" a “7. Muito Acima do Esperado”, e cujos dados estatísticos são apresentados na Tabela 6.

Tabela 6

Variáveis Desempenho Gerencial

\begin{tabular}{lcccc}
\hline \multicolumn{1}{c}{ Assertiva } & Variável & Média & Desvio-Padrão & Referencial \\
\hline Planejamento das atividades & DG1 & 4,56 & 1,32 & Malmi e Grandlund (2009) \\
\hline Controle dos Recursos & DG2 & 4,83 & 1,29 & Malmi e Grandlund (2009) \\
\hline Tomada de Decisão & DG3 & 4,96 & 1,20 & Malmi e Grandlund (2009) \\
\hline
\end{tabular}

Fonte: dados da pesquisa

\subsubsection{Desempenho Organizacional}

O desempenho organizacional medido por apenas uma variável, porém, é coerente com o propósito do estudo e do encadeamento das variáveis. Os respondentes foram solicitados a avaliar os gestores em relação ao atingimento da principal meta da organização, classificando o desempenho de 1. Muito Abaixo do Esperado a 7. Muito Acima do Esperado.

Tabela 7

Variável Desempenho Organizacional

\begin{tabular}{ccccc}
\hline Assertiva & Variável & Média & Desvio-Padrão & Referencial \\
\hline Atingimento da principal meta da organização & DO & 5,2 & 1,2 & $\begin{array}{l}\text { Anthony e Govindarajan } \\
\text { (2006) Crossan et al. (2009) }\end{array}$ \\
\hline
\end{tabular}

Fonte: dados da pesquisa 


\subsection{Modelagem de equação estrutural}

Utilizou-se a técnica de Modelagem de Equação Estrutural (MEE), apropriada para estimar uma série de relações de dependência inter-relacionadas simultaneamente (Hair Jr. et al, 2005), baseada em mínimos quadrados parciais, pois este método pode ser utilizado com amostras menores (Smith \& Langfield-Smith, 2004; Zwicker, Souza \& Bido, 2008).

\subsection{Procedimentos estatísticos}

A análise dos resultados baseou-se nas etapas recomendadas por Hair Jr et al. (2005), as quais incluem:

Etapa 1: Análise prévia dos 80 casos, buscando identificar casos não válidos, o que não foi identificado.

Etapa 2: Nesta etapa, avaliou-se o modelo de mensuração por meio da:

- Avaliação da correlação de cada indicador com seus respectivos construtos ou variáveis latentes, e pelo fato de que se trata de um estudo exploratório não foi excluída nenhuma variável, muito embora para dar maior robustez ao modelo essa correlação deveria ser superior a 0,7 consoante Hair Jr et. al. (2005).

- Análise da confiabilidade composta de cada construto, cujo índice deveria ser superior ou igual a 0,7, assim como Alpha de Cronbach, os quais têm a função de avaliar se o indicador mensura adequadamente o construto (Hair Jr. et al., 2005; Zwicker et al., 2008). Para construtos unidimensionais, esse procedimento não é necessário, que, no caso da pesquisa, o construto é Desempenho Organizacional, com Alpha de Cronbach igual a 1.

- Verificação da Variância Média Explicada (VME), que deveria ser superior a 50\% (Hair Jr. et al., 2005). A Variância Média Explicada é uma medida de validade convergente e reflete a quantia geral de variância nos indicadores explicada pelo construto latente. Neste estudo somente o construto Fontes de Conhecimento ficou com uma VME de 25\%, afetada pelo procedimento de não excluir variáveis com carga abaixo de 0,7 .

- Validade Discriminante, cujo teste é operacionalizado pela Raiz Quadrada da VME, a qual não pode ser menor que as correlações entre os construtos (Tenenhaus, Vinzi, Chatelini \& Lauro, 2005). O objetivo da validade discriminante é determinar se os indicadores têm uma relação mais forte com suas respectivas variáveis latentes do que com qualquer outra variável latente presente no modelo.

Etapa 3: Validação do Modelo Estrutural consistindo de:

- Verificação dos valores de $\mathrm{R}^{2}$, que significa a porcentagem de variância de uma variável latente que é explicada por outras variáveis latentes.

- Testar a hipótese de que os coeficientes da regressão sejam iguais a zero por meio do Teste $t$ de Student. Para um nível de significância de 0,05, o valor do t é de aproximadamente 1,96. O procedimento adotado foi utilizar a técnica bootstrapping, a qual consiste em um tipo de reamostragem aleatória com repetição (Hair Jr. et. al., 2005). Nessa pesquisa, foram utilizadas 200 reamostragens para o cálculo dos valores t.

O software utilizado para o teste foi o SmartPLS 2.0 M3 (Ringle, Wende, \& Will, 2005). 


\section{Apresentação e Discussão dos Resultados}

Na Figura 2 podem ser visualizados os relacionamentos entre os construtos e respectivas cargas fatoriais.

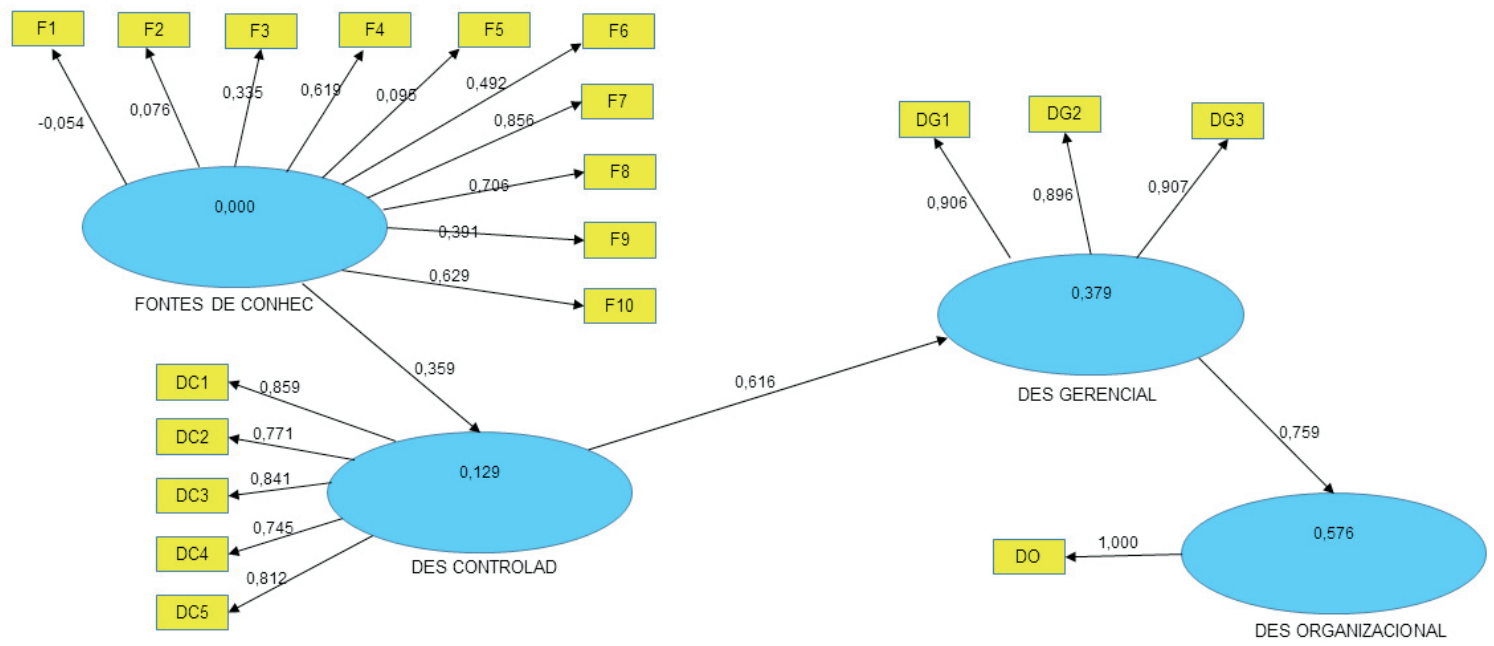

Fonte: SMARTPLS com base em dados da pesquisa

Figura 2. Modelo PLS dos relacionamentos estruturais

Na Tabela 8, podem ser visualizadas as estatísticas dos construtos.

Tabela 8

Estatísticas dos construtos

\begin{tabular}{llcccc}
\hline \multicolumn{1}{c}{ Construto } & \multicolumn{1}{c}{ Sigla } & VME & $\begin{array}{c}\text { Confiabilidade } \\
\text { Composta }\end{array}$ & R2 & $\begin{array}{c}\text { Alpha de } \\
\text { Cronbach }\end{array}$ \\
\hline Desempenho da Área de Controladoria & Des Controlad & 0,65 & 0,90 & 0,13 & 0,87 \\
\hline Desempenho Gerencial & Des Gerencial & 0,82 & 0,93 & 0,38 & 0,89 \\
\hline Desempenho Organizacional & Des Organizacional & 1,00 & 1,00 & 0,58 & 1,00 \\
\hline Fontes de Conhecimento & Fontes Conhec & 0,25 & 0,70 & - & 0,71 \\
\hline
\end{tabular}

Fonte: dados da pesquisa

Os resultados sugerem que apenas $13 \%$ das variações no desempenho de controladoria são explicadas pelas variações no construto fontes de conhecimento. Por um lado, isso sugere que podem existir outros fatores que explicam o desempenho da área de Controladoria, como qualidade de pessoal, infraestrutura (espaço físico e condições de trabalho, entre outras), tecnologia (computadores e sistemas de informações), cultura organizacional, entre outros. Oyadomari, Aguiar, Chen e Dultra-de-Lima (2013) desenvolvem uma análise mais aprofundada sobre esses dados.

Já o Desempenho Gerencial, medido pelas variáveis, planejamento, controle de recursos e tomada de decisões, tem $38 \%$ das variações explicadas pela variação no desempenho da Controladoria, o que pode ser considerado bastante representativo. Esses resultados sugerem a importância da Controladoria para obtenção de Desempenho Gerencial.

O alto poder de explicação do Desempenho Organizacional, com 58\% das variações explicadas pelas variações no Desempenho Gerencial, pode estar refletindo um elevado direcionamento das operacionalizações desses construtos, que são focadas em aspectos mais relacionados com o papel do Sistema de Controle Gerencial. Por outro lado, os resultados estão na mesma direção que indicam a importância das Capacidades Dinâmicas propostas por Teece, Pisano e Shuen (1997), e mais especificamente nos aspectos gerenciais ligados a planejamento, controle e decisões. 
A Tabela 9 apresenta as correlações entre as variáveis latentes e, na diagonal, em negrito, foram incluídos os valores da raiz quadrada da VME. Nesse caso, a raiz quadrada da VME sempre foi maior que as correlações, indicando validade discriminante.

Tabela 9

Matriz de Correlações e Raiz Quadrada da VME

\begin{tabular}{lcccc}
\multicolumn{1}{c}{ Variável latente } & $\mathbf{1}$ & $\mathbf{2}$ & $\mathbf{3}$ & $\mathbf{4}$ \\
\hline (1) Des Controlad & $\mathbf{0 , 8 1}$ & - & - & - \\
\hline (2) Des Gerencial & 0,62 & $\mathbf{0 , 9 0}$ & - & - \\
\hline (3) Des Organizacional & 0,60 & 0,76 & 1,00 & - \\
\hline (4) Fontes Conhec & 0,36 & 0,28 & 0,25 & 0,50 \\
\hline
\end{tabular}

Nota: Na diagonal, em negrito, foram incluídos os valores da raiz quadrada da VME.

Fonte: dados da pesquisa

Os testes estatísticos sobre os relacionamentos estruturais podem ser visualizados na Tabela 10.

Tabela 10

Coeficientes Estruturais do Modelo

\begin{tabular}{lccc}
\multicolumn{1}{c}{ Relacionamento Estrutural } & Coeficiente & Erro Padrão & Valor T \\
\hline Des Controlad $\Rightarrow$ Des Gerencial & 0,62 & 0,07 & 8,88 * \\
\hline Des Gerencial $\Rightarrow$ Des Organizacional & 0,76 & 0,07 & $10,477^{*}$ \\
\hline Fontes Conhec $\Rightarrow$ Des Controlad & 0,36 & 0,08 & 4,49 * \\
\hline
\end{tabular}

Nota: *estatisticamente significante ao nível de 5\%

Fonte: dados da pesquisa

Percebe-se, com base nos valores $\mathrm{T}$, que os três relacionamentos examinados neste estudo são significativos, considerando um nível de significância de 5\%. Dessa forma, não foi possível rejeitar as hipóteses, sendo aceito que:

H1: Grau de Obtenção de informações tem associação positiva com o Desempenho da Controladoria

H2: Desempenho da Controladoria tem associação positiva com Desempenho Gerencial

H3: Desempenho Gerencial tem associação positiva com o Desempenho Organizacional

Esses resultados sugerem que quanto maior a busca por conhecimento, maior será o desempenho da Controladoria. Isso indica que os profissionais que atuam nessa área devam se manter constantemente atualizados, particularmente em um momento caracterizado por alterações nas normas contábeis, as quais deverão influenciar as informações gerenciais fornecidas pela área de Controladoria, uma vez que é tênue a fronteira entre Contabilidade Financeira e Contabilidade Gerencial.

Os resultados são importantes e sugerem que a educação continuada dos profissionais de controladoria caracterizada pelas variáveis livros, artigos acadêmicos, seminários e cursos tem contribuído para melhorar o desempenho da controladoria, o que é mais um argumento para que as políticas de desenvolvimento desses profissionais incentivem a educação continuada. Outro fator importante é que a busca de conhecimento também se faz por meio de participação em reuniões de associações empresariais e profissionais, o que destaca o papel que associações como Conselho Regional de Contabilidade, Associação Nacional de Executivos de Finanças e Contabilidade (Anefac) e entidades empresariais possuem na disseminação de conhecimento 
entre os profissionais de controladoria. Por outro lado, apesar da média alta, a busca de informações em outros departamentos apresentou alta variabilidade. Portanto, torna-se necessário estimular essa busca de informação entre as áreas, uma vez que podem contribuir para gerar conhecimento tácito.

Quanto ao Desempenho da Controladoria, as notas altas evidenciam que esses atributos estão com bom desempenho, indicando que Controladoria atende os usuários de forma bastante satisfatória.

Os resultados também sugerem que há associação positiva entre Desempenho da Controladoria e Desempenho Gerencial, indicando que uma boa atuação da Controladoria implica positivamente melhor planejamento das atividades, controle dos recursos e tomada de decisões por parte dos gestores. Essas variáveis são bastante influenciadas por uma área de Controladoria que forneça informações no tempo adequado e com boa qualidade, e que faça isso utilizando recursos dentro do orçamento, desenvolvendo projetos de melhoria dentro da sua própria área, obtendo assim um elevado grau de satisfação dos usuários internos.

Por fim, há associação positiva entre Desempenho Gerencial e Desempenho Organizacional, este último medido pelo grau de atingimento da principal meta da organização. Este resultado, por um lado, pode estar sendo afetado pelo ambiente econômico favorável que permeia o período em que a pesquisa foi realizada, mas por outro lado pode indicar a importância de um gestor planejar as atividades, controlar os recursos e tomar decisões, suportado por um adequado e ágil sistema de informações e que atenda às necessidades gerenciais.

Resumidamente, os resultados sugerem que uma área de Controladoria que busca conhecimento nas fontes internas e externas consegue gerar informações com boa qualidade e no tempo adequado, a um custo compatível, auxiliada pela gestão de projetos departamentais, consegue atender às expectativas dos usuários. Esses gestores, por sua vez, suportados por uma Controladoria eficaz e eficiente, planejam, controlam e tomam decisões mais assertivas, contribuindo para que a organização atinja satisfatoriamente o seu principal objetivo.

Os resultados estão em consonância com a preocupação de identificar o papel da área de Controladoria, e confirma que o Sistema de Controle, junto com outro recurso, no caso o Desempenho Gerencial, pode ser considerado como um recurso importante para obtenção de vantagem competitiva consoante Barney (2007). Também se junta aos estudos que tentam identificar os efeitos de uma gestão de boas práticas de Controladoria, identificando os efeitos desta para a organização, como os estudos de Tsui (2001), Agbejule e Saarikoski (2006) e Weißenberger e Angelkort (2011).

Ao mesmo tempo, os resultados da pesquisa contribuem com a literatura nacional e internacional ao estudar especificamente o construto Desempenho da Controladoria e relacionar com o Desempenho Gerencial, que parece ser o relacionamento mais próximo dado que a Controladoria gera informações para os gestores planejarem, executarem e controlarem os recursos e ações.

\section{Considerações Finais}

Este estudo identificou se existem associações entre a busca de conhecimento, desempenho da controladoria, desempenho gerencial e desempenho organizacional, um tema que tem como pano de fundo os interesses dos práticos em identificar as chamadas melhores práticas, interesse este que também é o propósito da criação da Teoria em Contabilidade Gerencial.

O estudo avança ao propor um construto de Desempenho da Controladoria, além de ser uma adaptação de construto de Desempenho Gerencial e de Desempenho Organizacional, mais relacionado com o papel da Controladoria. Os resultados indicaram que, para 80 profissionais de controladoria que atuam em empresas brasileiras, existem associações positivas entre a busca de conhecimento, desempenho da área de Controladoria, desempenho gerencial e desempenho organizacional, com esses relacionamentos encadeados com associação positiva e com significância estatística.

Os resultados apontam para contribuições para a prática, indicando que esses profissionais devem continuar envidando esforços em educação continuada, e que ainda há oportunidade para se buscar 
conhecimento dentro da organização. Esses esforços conjugam para a obtenção de desempenho da área de Controladoria, e que esta é importante para que os gestores planejem, controlem e tomem decisões de forma adequada, e que isso contribui positivamente para o atingimento do principal objetivo da organização.

Para a literatura, entende-se que a pesquisa contribui ao confirmar empiricamente o entendimento da área de Controladoria como um recurso valioso, validando o preconizado pelos livros-textos. Essa área pode ser entendia como uma geradora de capacidades dinâmicas, principalmente adaptando mecanismos de mensuração e instrumentos de avaliação de desempenho aderentes à realidade organizacional e aos objetivos estratégicos da empresa.

Para a comunidade científica, os resultados podem ser úteis para, ainda que inicialmente, mostrar que as fontes de informações mais utilizadas por esses profissionais não são os artigos acadêmicos, mas, sim, informações obtidas pelo processo de socialização por meio de rede de contatos e associações profissionais, o que pode ser visto como um espaço para que acadêmicos possam interagir com os profissionais da prática e assim criar conhecimento.

Ao mesmo tempo, os resultados devem ser vistos com cuidado, pois a percepção de desempenho foi feita com base na opinião de profissionais da área de Controladoria. Assim outros estudos que capturem a dimensão desempenho da área de Controladoria pela percepção dos usuários da Controladoria poderiam ser desenvolvidos para evitar esse viés.

Muito embora os resultados não possam ser generalizados, eles podem ser considerados embriões de futuras pesquisas que identifiquem, por meio de estudos de casos, como a área de Controladoria cria e compartilha conhecimento, e que identifiquem a contribuição da área de Controladoria aos gestores organizacionais em seu trabalho rotineiro de planejamento, controle e tomada de decisão.

\section{Referências}

Agbejule, A., \& Saarikoski, L. (2006) The effect of cost management knowledge on the relationship between budgetary participation and managerial performance. The British Accounting Review, 38(4), pp.427-440.

Anthony, R. N., \& Govindarajan, V. (2006). Sistemas de Controle Gerencial. São Paulo: Atlas.

Baldvinsdottir, G., Mitchell, F., \& Nørreklit, H. (2010) Issues in the relationship between theory and practice in management accounting. Management Accounting Research, 21(2), pp.79-82.

Bardin, L. (2004) Análise de conteúdo (3 $3^{\text {rd }}$ d.). Lisboa: Edições 70.

Barney, J. (2007) Gaining and sustaining competitive advantage ( $3^{\text {rd }}$ ed.) Upper Saddle River, NJ: Pearson Education.

Barney, J. B. (2001) Resource-based theories of competitive advantage: a ten-year retrospective on the resource-based view. Journal of Management, 27(6), pp.643-650. doi:10.1177/014920630102700602

Borinelli, M. L.(2006). Estrutura conceitual básica de controladoria : sistematização à luz da teoria e da práxis Tese (Doutorado em Ciências Contábeis). Programa de Pós-Graduação em Ciências Contábeis. Departamento de Contabilidade e Atuária - FEA - São Paulo, SP, Brasil.

Cadez, S., \& Guilding, C. (2008). An exploratory investigation of an integrated contingency model of strategic management accounting. Accounting, Organizations and Society, 33(7-8), 836-863.

Calhoun, M. A., \& Starbuck, W. H. (2003) Barriers to Creating Knowledge. In: M. Easterby-Smith, \& M. A. Lyles (Eds.) The Blackwell handbook of organizational learning and knowledge management. Oxford: Blackwell, pp.473-492.

Catelli, A. (1999) Controladoria: uma abordagem da gestão econômica - GECON. São Paulo: Atlas.

Chenhall, R. H., \& Langfield-Smith, K. (2007) Multiple perspectives of performance measures. European Management Journal, 25(4), pp. 266-282. 
Craik, K. H., Ware, A. P., Kamp, J., O’Reilly, C., Staw, B., \& Zedeck, A. (2002) Exploration of construct validity in a combined managerial and personality assessment programme. Journal of Occupational and Organizational Psychology, 75, pp.171-193. doi: 10.1348/09631790260098758

Crossan, M. M., Rouse, M. J., Fry, J.N., \& Killing, J.P. (2009) Strategic analysis and action. Toronto, Ontario: Pearson.

Dillman, D. (2000) Constructing the questionnaire. Mail and internet surveys. New York: John Wiley \& Sons.

Donaldson, L. (2001) The contingency theory of organizations. Thousand Oaks, CA: Sage Publications.

Flick, U. (2009) Introdução à pesquisa qualitativa (3a ed). Porto Alegre: Bookman.

Frezatti, F., Aguiar, A. B., \& Guerreiro, R. (2007) Diferenciações entre a contabilidade financeira e a contabilidade gerencial: uma pesquisa empírica a partir de pesquisadores de vários países. Revista Contabilidade e Finanças, 18(44), pp. 9-22.

Garrison, .R., Noreen, E.W.; \& Brewer, P.C. (2007). Contabilidade gerencial. 11. ed Rio de Janeiro: LTC.

Grant, R. M. (1991) The resource-based theory of competitive advantage: implications for strategy formulation. California Management Review, Spring, 34(1), pp. 114-135,

Hair Jr., J. F., Anderson, R. E., \& Black, W. (2005) Análise multivariada de dados. Porto Alegre: Bookman.

Hall, M. (2010) Accounting information and managerial work. Accounting, Organizations and Society, 35(3), pp. 301-315.

Henri, J. F. (2006) Management control systems and strategy: a resource-based perspective. Accounting, Organizations and Society, 31(6), pp.529-558.

Hitt, M. A., Ireland, R. D., \& Hoskisson, R. E. (2001) Strategic Management: competitiveness and globalization (concepts). Thomson Learning.

Hunton, J. E., Wier, B., \& Stone, D.N. (2000) Succeeding in managerial accounting. Part 2: knowledge, ability, and rank. Accounting, Organizations and Society, 25(2), pp.751-762.

Lunkes, R. J., Schnorrenberger, D., \& Rosa, F. S. (2013). Funções da Controladoria: uma análise no cenário brasileiro. Revista Brasileira de Gestão de Negócios, 15(47), pp.283-299.

Mahoney, T. A., Jerdee. T. H. \& Carroll, S. J. (1965). The Jobs of Management. Industrial Relations, 4(2), pp. 97-1 10. DOI: 10.1111/j.1468-232X.1965.tb00922.x

Malmi, T., \& Grandlund, M. (2009) In search of management accounting theory. European Accounting Review, 18 (3), pp.597-620.

Martins, J. T., Quincozes, E. R. F., Pereira, M. F., Fialho, F.A.P. (2009). A contribuição das redes sociais para o desenvolvimento de projetos de pesquisa, desenvolvimento e inovação (P,D\&I): o caso da Embrapa. Simpósio de Excelência em Gestão e Tecnologia. Recuperado em 15/10/2013 de www.aedb.br/seget/ artigos09/290_artigo.pdf.

Mol, M. J., \& Birkinshaw, J. (2009) The sources of management innovation: When firms introduce new management practices. Journal of Business Research, 62(12), pp.1269-1280.

Morris, T., \& Empson, L. (1998) Organization and Expertise: an exploration of knowledge bases and the management of accounting and consulting firms. Accounting, Organizations and Society, 23 (5-6), pp. 609-624.

Mouritsen, J. (1996) Five aspects of accounting departments' work. Management Accounting Research, 7(3), pp. 283-303.

Nonaka, I., \& Takeuchi, H. (1997) Criação de Conhecimento na Empresa: Como as Empresas Japonesas Geram a Dinâmica da Inovação. Rio de Janeiro: Elsevier. Oyadomari, J.C.T; Aguiar, A.B. Chen, Y-T. 
Dultra-de-Lima, R.G. (2013) Inovação de práticas de controle gerencial e fontes de informação. Revista Contabilidade e Organizações, 19, pp.17-26

Pettigrew, A., \& Massini, S. (2003) Innovative forms of organizing: trends in Europe, Japan and the USA in the 1990s. In A. Pettigrew, R. Whittington, L. Melin, C. Sánchez-Runde, F. van den Bosch, W. Ruigrok, \& T. Numagami (Eds.), Innovative forms of organizing: International Perspectives. London: Sage. pp. 1-33.

Ramírez, A. M., Morales, V. J. G., Rojas, R. M., (2011). Knowledge Creation, Organizational Learning and Their Effects on Organizational Performance. Engineering Economics,22(3), pp. 309-318.

Ray, G., Barney, J. B., \& Muhanna, W. A. (2004) Capabilities, Business Processes, and Competitive Advantage: Choosing the Dependent Variable in Empirical Tests of the Resource-Based View. Strategic Management Journal, 25(1), pp. 23-37. DOI: 10.1002/smj.366.

Ringle, C. M., Wende, S.\& Will, A. (2005) Smartpls 2.0 M3 (Beta). Germany: University of Hamburg. Recuperado em 15 outubro, 2013, de <http://www.smartpls.de>.

Robertson, I.T., Baron, H., Gibbons, P., Maciver, R. \& Nyfield, G. (2000) Concientiousness and managerial performance. Journal of Occupational and Organizational Psychology, 73(2), pp.171180. doi: $10.1348 / 096317900166967$

Scapens, R. W. (1994) Never Mind the Gap: towards an institutional perspective on management accounting practice. Management Accounting Research, 5(3-4), pp.301-321.

Simons, R. (2000). Performance Measurement \& Control Systems for Implementing Strategy. Prentice Hall Upper Saddle River NJ, pp. 77-109.

Smith, D., \& Langfield-Smith, K. (2004) Structural equation modeling in management accounting research: critical analysis and opportunities. Journal of Accounting Literature, 23, pp.49-86.

Teece, D. J., Pisano, G. \& Shuen, A. (1997) Dynamic capabilities and strategic management. Strategic Management Journal, 18(7), pp.509-533.

Tenenhaus, M., Vinzi, V. E., Chatelin, Y.-M., \& Lauro, C. (2005) PLS path modelling. Computational Statistics and Data Analysis, 48(1), pp.159-205.

Tsui, J. S. L. (2001) The impact of culture on the relationship between budgetary participation, management accounting systems, and managerial performance: an analysis of Chinese and Western managers. The International Journal of Accounting, 36(2), pp.125-146.

Van Helden, G. J., Aardema, H., Ter Bogt, H. J., \& Groot, T. L. C. M. (2010) Knowledge creation for practice in public sector management accounting by consultants and academics: Preliminary findings and directions for future research. Management Accounting Research, 21(2), pp. 83-94.

Vera-Muñoz, S. C., Ho, J. L., \& Chow, C. W. (2006) Enhancing Knowledge Sharing in Public Accounting Firms. Accounting Horizons, 20 (2), pp. 133-155.

Weißenberger, B.E., \& Angelkort, H. (2011). Integration of financial and management accounting systems: The mediating influence of a consistent financial language on controllership effectiveness. Management Accounting Research, 22(3), pp.160-180.

Zwicker, R., Souza, C. A., \& Bido, D. S. (2008) Uma revisão do Modelo do Grau de Informatização de Empresas: novas propostas de estimação e modelagem usando PLS (partial least squares). Anais do XXXII Encontro da ANPAD, Rio de Janeiro, RJ, Brasil. 